REVISTA CIENCIAS BIOMÉ DICAS

PRESENTACIÓN DE CASOS CLÍNICOS

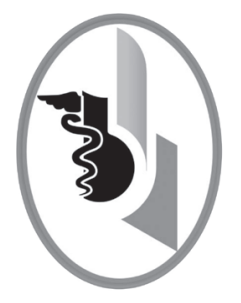

\title{
TUMOR GLIONEURONAL PAPILAR EN FOSA POSTERIOR
}

PAPILLARY GLIONEURONAL TUMOR OF THE POSTERIOR FOSSA

Tuñón-Pitalúa Martha Cecilia ${ }^{1}$

Ruiz-Cáez Karina María ${ }^{1}$

Niño-Hernández Lucía Mercedes ${ }^{1}$

Benedetti-Isaac Juan Carlos ${ }^{2}$

Alcalá-Cerra Gabriel ${ }^{3}$

Remolina-López Anderson Julián ${ }^{3}$

Correspondencia: mtunon@gmail.com

Recibido para evaluación: junio-17-2014. Aceptado para publicación: mayo-22-2015.

\section{RESUMEN}

Introducción: el tumor glioneuronal papilar (TGNP) es una entidad rara y de reciente tipificación. De probable origen en las células pluripotenciales de la placa subependimaria, que está ubicada principalmente alrededor de las astas temporales de los ventrículos laterales. En dichos sitios típicamente se presentan estas neoplasias.

Caso clínico: se presenta un caso atípico de TGNP en una paciente de 48 años de edad, con tumor que comprometía el vermis inferior y ambos hemisferios cerebelosos.

Conclusión: el TGNP se ubica generalmente en el espacio supratentorial. No obstante, se ha señalado la existencia de células pluripotenciales en la placa subependimaria, alrededor del área postrema, en la eminencia media del piso del cuarto ventrículo y en el plexo coroideo, ello pudiese explicar la exótica ocurrencia de TGNP en la fosa posterior. Rev.cienc.biomed. 2015;6(1):150-154

\section{PALABRAS CLAVE}

Neurociencias; Sistema nervioso central; Neoplasias encefálicas

\section{SUMMARY}

Introduction: the papillary glioneuronal tumor (PGT) is an uncommon disease and it has recent categorization. It has probable origin in the pluripotent stem cells of the subependymal zone that is located mainly around the temporal horns of the lateral ventricles. In these zones typically are presented these neoplasms.

Case record: an atypical case of PGT is presented in a patient with 48 years old, in who the tumor compromised the inferior cerebellar vermis and both cerebellum hemispheres. Conclusion: the PGT is generally located in the supratentorial area. However, it has been indicated the existence of pluripotent stem cells of the subependymal zone, around the area postrema, at the median eminence in the floor of the fourth ventricle and in the choroid plexus, which could explain the exotic occurrence of the PGT in the posterior fossa Rev.cienc.biomed. 2015;6(1):150-154

\section{KEYWORDS}

Neurosciences; Central nervous system; Brain neoplasms.

\footnotetext{
Médico. Sección de Patología. Facultad de Medicina. Universidad de Cartagena. Cartagena. Colombia.

Médico. Servicio de Neurocirugía. Fundación Centro Colombiano de Epilepsia y Enfermedades Neurológicas - FIRE. Cartagena. Colombia.

Médico. Sección de Neurocirugía. Facultad de Medicina. Universidad de Cartagena. Cartagena. Colombia.
} 
ISSN: 2215-7840, 6(1), enero-junio 2015, Tunón-Pitalúa Martha Cecilia, Ruiz-Cáez Karina María, Niño-Hernández Lucía Mercedes, Benedetti-Isaac Juan Carlos, Alcalá-Cerra Gabriel, Remolina-López Anderson Julián

\section{INTRODUCCIÓN}

El tumor glioneuronal papilar (TGNP) es una entidad rara, de reciente tipificación, incluida desde 2007 en el grupo de tumores glioneuronales mixtos (1). Durante varios años fue confundido con los gangliogliomas, sin embargo actualmente se reconoce como una entidad morfológica e inmunofenotípicamente diferente.

De los 60 casos reportados de TGNP hasta 2011, la mayoría habían sido descritos alrededor de los ventrículos laterales, principalmente bordeando las astas temporales (2). Aunque su localización es casi exclusiva del espacio supratentorial, existe reporte de una lesión ubicada en la fosa posterior, adyacente a la pared posterior del cuarto ventrículo (3). El objetivo es presentar el caso de una paciente con un TGNP en el área del vermis inferior y en relación con ambos hemisferios cerebelosos. Se revisan las publicaciones referentes a estas lesiones, con especial énfasis en las teorías que permiten explicar su génesis en el interior de la fosa posterior.

\section{CASO CLÍNICO}

Paciente femenino de 48 años de edad con cefalea de tres meses de evolución, de intensidad moderada y refractaria al tratamiento médico. En el momento de su ingreso, el examen neurológico fue normal.

Se realizó resonancia magnética contrastada con gadolinio que demostró una lesión intraaxial que comprometía el vermis inferior y el área posteromedial de ambos hemisferios cerebelosos, extendiéndose en el plano sagital en sentido caudal hasta el nivel del foramen magno. No se observó edema alrededor de la lesión ni deformidad por efecto de masa sobre el cuarto ventrículo o el tronco encefálico. La señal de la lesión era heterogénea, predominantemente baja en las secuencias de $T 1$, entremezcladas con zonas de alta señal en las secuencias de T2. La captación del medio de contraste también era heterogénea y especialmente intensa en la periferia de la lesión (Figura $\mathrm{N}^{0} 1$ ).
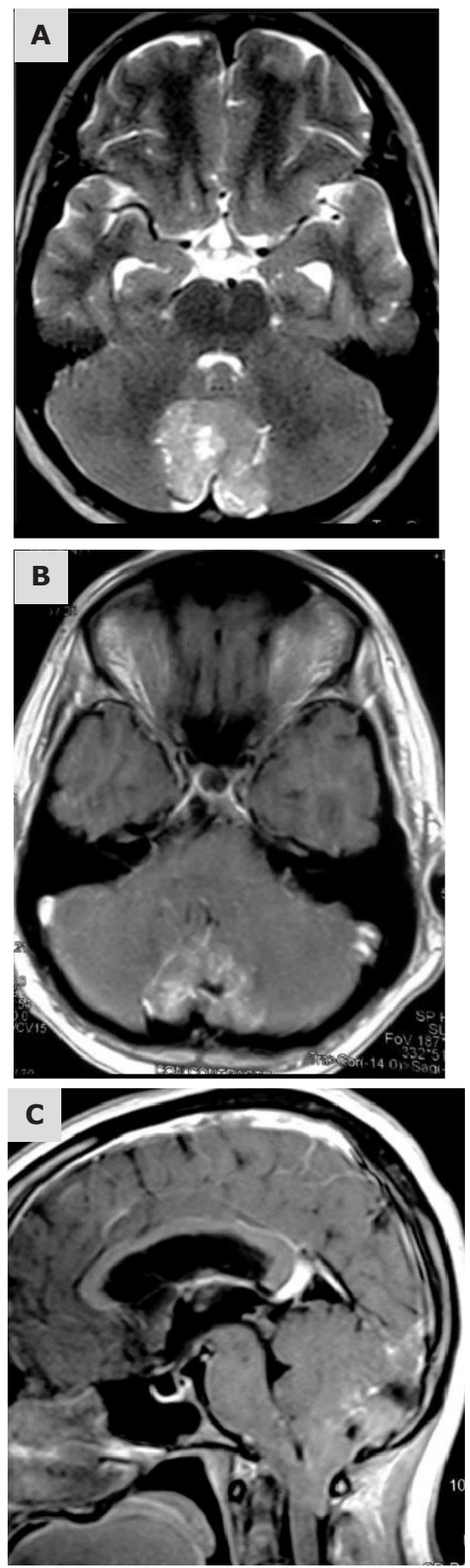

Figura No 1.

[A]. Resonancia magnética contrastada. Secuencias T2 axiales, muestra baja señal excepto en su centro, donde existe un pequeño componente quístico. Sin edema perilesional, ausencia de efecto de masa.

[B]. Secuencia T1 axial contrastada, muestra neoplasia intraxial que compromete el vermis y parte de la región medial de ambos hemisferios cerebelosos. Moderado realce con el contraste con zona central hipointensa.

[C]. Secuencia T1 axial contrastado, el tumor vermiano se extiende en sentido caudal hacia el foramen magno, situándose por detrás de la unión bulbo medular. 
Los estudios histopatológicos revelaron lesión tumoral constituida por estructuras papilares tapizadas por células cuboidales en monocapa, sobre un tallo ricamente vascularizado con marcada hialinización. Lo anterior estaba asociado a áreas sólidas de fondo fibrilar con células intermedias o grandes de apariencia neuronal. La inmunohistoquímica mostró inmunoreactividad fuerte y difusa para proteína ácida fibrilar glial e inmunoreactividad dual para sinaptofisina, predominante en las áreas sólidas (Figura No 2 y No 3 ).
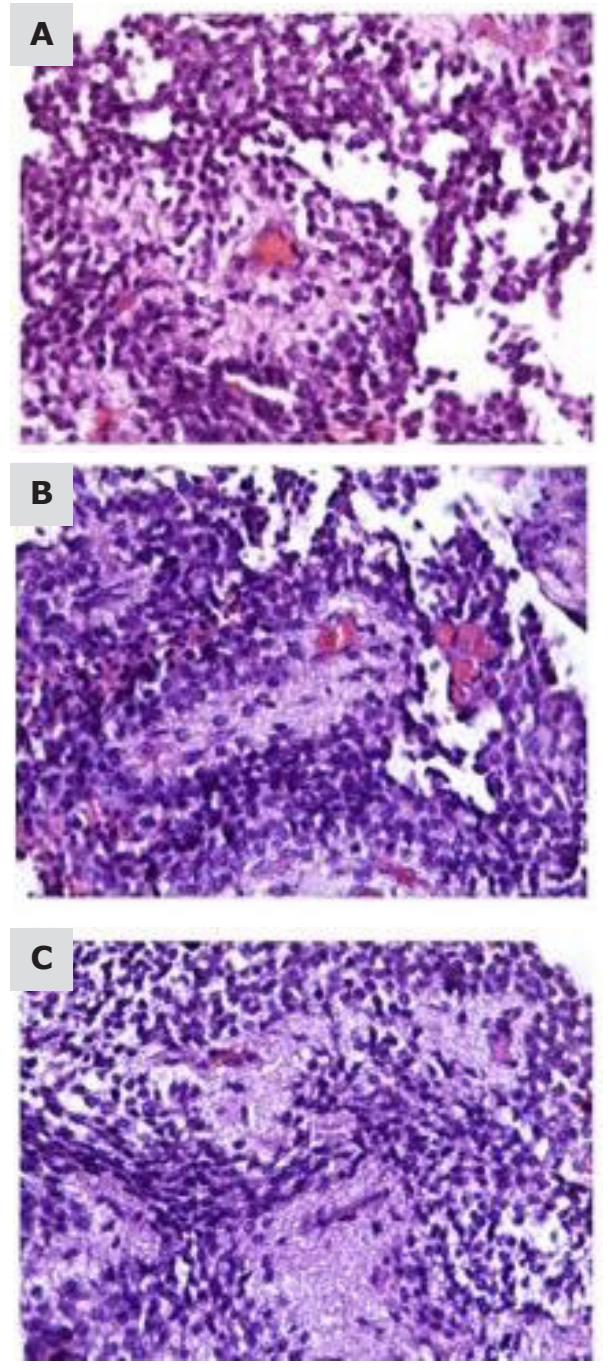

Figura $\mathbf{N}^{\circ} 2$.

[A]. H\&E (40x). Población monótona de células pequeñas redondas sin atipia, ni mitosis en un fondo fibrilar [B]. H\&E (40x). Estructura papilar tapizada en seudocapa.

[C]. H\&E (20x). Formación de estructuras papilares sobre un corte vascularizado, tapizadas por células monótonas seudoestratificadas, que en áreas adoptan un patrón sólido.
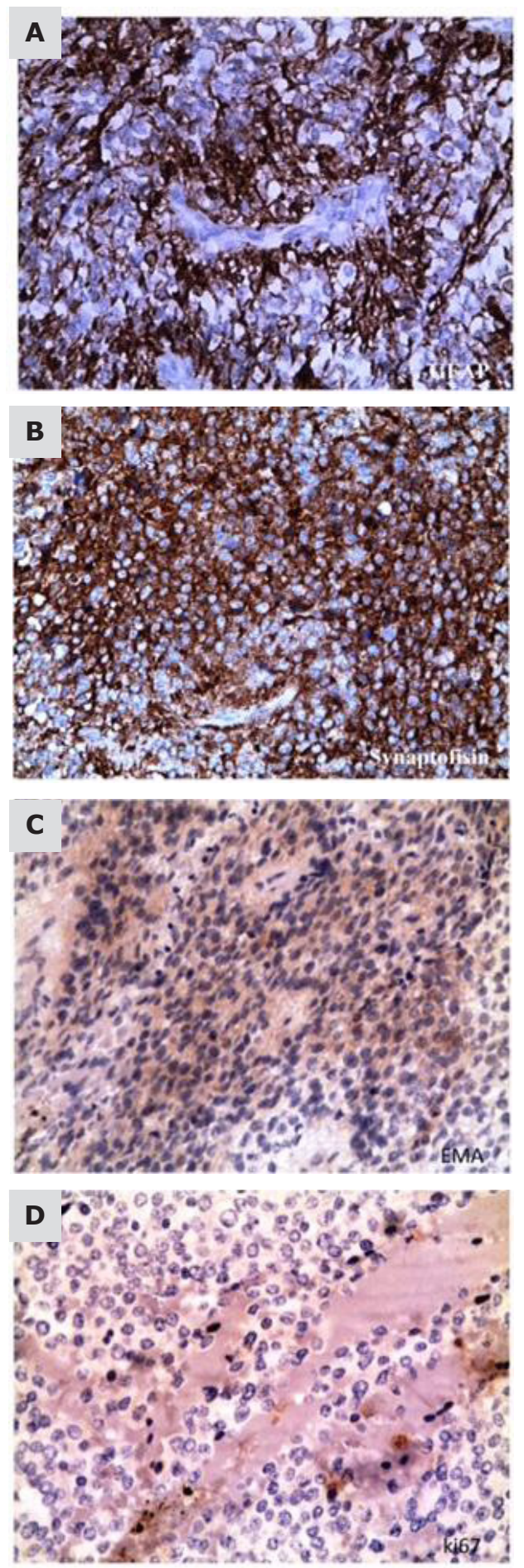

Figura No 3.

[A]. Proteína ácida fibrilar glial (GFAP) (40x). Inmunoreactividad fuerte y difusa en la población de células pequeñas redondas

[B]. Sinaptofisina (40x). Inmunoreactividad dual en algunas de las células con predominio por las áreas solidas

[C]. EMA (20x). Inmunoreactividad focal en algunas de las células

[D]. Ki 67 bajo. (20X). 
ISSN: 2215-7840, 6(1), enero-junio 2015, Tuñon-Pitalúa Martha Cecilia, Ruiz-Cáez Karina María, Niño-Hernández Lucía Mercedes, Benedetti-Isaac Juan Carlos, Alcalá-Cerra Gabriel, Remolina-López Anderson Julián

Se realizó resección total mediante craniectomía suboccipital mediana. La evolución postoperatoria tras doce meses de seguimiento fue adecuada y los controles imagenológicos no han demostrado recidiva tumoral.

\section{DISCUSIÓN}

El TGNP es una neoplasia circunscrita, clínica y biológicamente indolente, constituida por una población neoplásica bifásica de astrocitos que adoptan disposición pseudopapilar, entremezclado con colecciones neuronales (1).

Los datos epidemiológicos referentes a la entidad son escasos y derivados exclusivamente del análisis de reportes de casos aislados. Se ha establecido que la mayoría han sido identificados en adultos jóvenes, con una media de 23.6 años al momento del diagnóstico $(4)$ y sin predilección por género $(5,6)$.

Los reportes publicados se han enfocado en describir detalladamente los hallazgos histopatológicos y pocos a las características radiológicas, tal como fue puntualizado por Xiao (5) y Amamiya (7) en dos revisiones, donde además se destacada la escasez de información acerca de los hallazgos en las imágenes de resonancia magnética.

La mayoría de estos tumores han sido encontrados como masas voluminosas predominantemente quísticas y bien delimitadas, con un nódulo mural que capta intensamente el medio de contraste. Estos hallazgos en conjunto han sido descritos en el $90.0 \%$ de los informes, mientras que en solo $8.6 \%$ el componente sólido constituye el mayor volumen tumoral, tal como se observó en el presente caso (5). Adicionalmente pueden encontrarse calcificaciones, las cuales se describen en el $25.0 \%$ de los casos (6).

Los TGNP se han descrito casi exclusivamente al interior de los hemisferios cerebrales, sin embargo está reportado el caso de un paciente portador de un tumor que comprometía el vermis inferior derecho e infiltraba el agujero de Luschka ipsilateral (3). Las relaciones anatómicas en el presente caso son muy similares, sugiriendo la existencia de un origen común en ambas lesiones. Bennett y colaboradores demostraron sitios específicos a lo largo del tercer y cuarto ventrículo, conocidos como órganos circunventriculares, los cuales contienen células que expresan proteínas de filamentos intermedios (nestina, vimentina, GFAP) y factores de transcripción (Sox2). Los órganos circuventriculares contienen células pluripotenciales/progenitoras del neuroepitelio embrionario. Se han señalado en la zona subventricular de la pared anterolateral del epéndimo y en la zona subgranular del giro dentado hipocampal $(8,9)$.

En cultivos, dichas células pueden diferenciarse de neuronas o glía, según el tratamiento exógeno que reciban. La presencia de estas células en el área postrema, la eminencia media del cuarto ventrículo y el plexo coroideo puede explicar la génesis de los TGNP dentro del espacio infratentorial (8).

La histogénesis del TGNP no ha sido esclarecida. La diferenciación retrógrada de las células maduras para producir tumores de linaje mixto, glial y neuronal, parece poco probable. La localización periventricular, encontrada en $78.4 \%$ de los casos (6), así como la diferenciación dual, sugiere que estos tumores se originarían de las células madre bipotenciales neurogliales de la zona germinativa de la placa subependimaria (3), las cuales son especialmente abundantes alrededor de los ventrículos laterales en mamíferos (10).

Adicionalmente, los estudios moleculares han demostrado que las células de los TGNP expresan PDGF, Olig2, nestina, HNK-1 y NCAM-L, las cuales también son exhibidas por las células progenitoras bipotenciales neurogliales (4).

Histopatológicamente, el TGNP se caracteriza por un patrón bifásico constituido por seudopapilas y áreas solidas $(1,11)$. Las papilas se encuentran tapizadas por células gliales de forma cuboidal de núcleo redondo y escaso citoplasma, distribuidos en monocapa o seudoestratificadas, descansando sobre un centro vascular hialinizado $(1,2)$. Las áreas sólidas se encuentran constituidas por elementos neuronales pequeños, intermedios o grandes, apoyados sobre una matriz fibrilar (12). El parénquima cerebral que circunda al tumor usualmente presenta cambios reactivos incluyendo fibras de Rosenthal $(10,12)$, astrocitos con prominentes glóbulos granula- 
res eosinofílicos $(10,13)$, calcificaciones, células espumosas, hemosiderófagos $(10,14)$, degeneración quística focal, cambios vasculares angiomatoides y componentes minigemistocíticos $(14,15)$.

Los hallazgos inmunohistoquímicos dependerán del componente tumoral. En las áreas neuronales se evidencia positividad para sinaptofisina, enolasa neuroespecífica, B-tubulina clase-III y Neu-N; mientras que las gliales son positivas para GFAP y S-100. El Ki-67 o el MIB-1 generalmente es menor del 3\% (2), a excepción de la variante minigemistocítica que ha demostrado tener una mayor actividad proliferativa y un índice mayor del 10\% (14).

La modalidad terapéutica de elección para los TGNP es la resección macroscópica total. Como la mayoría de los tumores clasificados como grado-I, la tasa de recidivas es extremadamente baja. El valor de la quimioterapia y/o radioterapia adyuvante es incierto y estas modalidades solo han sido utilizadas en algunos casos que presentaron altos índices de proliferación o cuando solo fueron resecados parcialmente. Aun así, el pronóstico generalmente es favorable y la tasa de recurrencias aún en los casos atípicos es muy baja (4).

\section{CONCLUSIÓN}

El TGNP es un raro tumor mixto glioneuronal localizado casi exclusivamente a nivel supratentorial, adyacente a los ventrículos laterales; inmiscuido al interior de los lóbulos frontales, temporales y/o parietales, pero también se puede encontrar ocasionalmente alrededor del área postrema, la eminencia media del piso del cuarto ventrículo y el plexo coroideo.

CONFLICTO DE INTERESES: ninguno que declarar.

FINANCIACIÓN: recursos propios de los autores. Estudios de laboratorio, insumos hospitalarios, medicamentos y honorarios profesionales aportados dentro de la atención asistencial.

\section{REFERENCIAS BIBLIOGRÁFICAS}

1. Louis DN, Ohgaki H, Wiestler OD, Cavenee WK, Burger PC, Jouvet A, et al. The 2007 WHO classification of tumours of the central nervous system. Acta Neuropathol. 2007;114:97-109.

2. Allende DS, Prayson RA. The expanding family of glioneuronal tumors. Adv Anat Pathol. 2009;16:339.

3. Ulivieri S, Oliveri G, Cerase A, Miracco C. Papillary glioneuronal tumor of the fourth ventricle: case report and review of the literature. G Chir. 2010;31:368-70.

4. Govindan A, Mahadevan A, Bhat DI, Arivazhagan A, Chakraborti S, Suja MS, et al. Papillary glioneuronal tumor-evidence of stem cell origin with biphenotypic differentiation. J Neurooncol. 2009;95:7180.

5. Xiao H, Ma L, Lou X, Gui Q. Papillary glioneuronal tumor: radiological evidence of a newly established tumor entity. J Neuroimaging. 2011;21:297-302.

6. Myung JK, Byeon S, Kim B, Suh J, Kim SK, Park CK, et al. Papillary glioneuronal tumors: A review of clinicopathologic and molecular genetic studies. Am J Surg Pathol. 2011;35:1794-805.

7. Amemiya S, Shibahara J, Aoki S, Takao H, Ohtomo K. Recently established entities of central nervous system tumors: review of radiological findings. J Comput Assist Tomogr. 2008;32:279-85.

8. Bennett L, Yang M, Enikolopov G, Iacovitti L. Circumventricular organs: A novel site of neural stem cells in the adult brain. Mol Cell Neurosci. 2009;41:337-47.

9. Mignone JL, Kukekov V, Chiang AS, Steindler D, Enikolopov G. Neural stem and progenitor cells in nestin-GFP transgenic mice. J Comp Neurol. 2004;469:311-24.

10. Komori T, Scheithauer BW, Anthony DC, Rosenblum MK, McLendon RE, Scott RM, et al. Papillary glioneuronal tumor: a new variant of mixed neuronal-glial neoplasm. Am J Surg Pathol. 1998;22:117183.

11. Prayson RA. Papillary glioneuronal tumor. Arch Pathol Lab Med. 2000;124:1820-3.

12. Radotra BD, Kumar Y, Bhatia A, Mohindra S. Papillary glioneuronal tumor: a new entity awaiting inclusion in WHO classification. Diagn Pathol. 2007;2:6.

13. Guo SP, Zhang F, Li QL, Li Q, Wang WL, Li FF. Papillary glioneuronal tumor-contribution to a new tumor entity and literature review. Clin Neuropathol. 2008;27:72-7.

14. Ishizawa T, Komori T, Shibahara J, Ishizawa K, Adachi J, Nishikawa R, et al. Papillary glioneuronal tumor with minigemistocytic components and increased proliferative activity. Hum Pathol. $2006 ; 37: 627-30$.

15. Tsukayama C, Arakawa Y. A papillary glioneuronal tumor arising in an elderly woman: A case report. Brain Tumor Pathol. 2002;19:35-9. 